\title{
ON THE TRANSPORT OF CHARGED PARTICLES IN TURBULENT FIELDS: COMPARISON OF AN EXACT SOLUTION WITH THE QUASILINEAR APPROXIMATION
}

\author{
J. R. JoKIPII* \\ Downs Laboratory of Physics, California Institute of Technology, \\ Pasadena, Calif. 91109 , U.S.A. \\ and \\ I. LERCHE \\ Enrico Fermi Institute, University of Chicago, Chicago, Ill., U.S.A.
}

(Received 14 November 1972)

\begin{abstract}
Absiract-We set up and solve, the problem of charged-particle transport in a magnetic field which is solely a function of time. The solution is obtained exactly, to all orders in the field, in the limit of large wavelengths normal to the magnetic field. We show that the usual quasilinear, Fokker-Planck approximation is equal to the exact solution in the limit of times large compared with the correlation time of the fluctuating field. This is just the regime where the approximation has been used in the past, and this special case thus gives some support to the standard approximation techniques.
\end{abstract}

\section{INTRODUCTION}

THE PROBLEM of the propagation of charged particles in random fields is of fundamental importance to modern astrophysics, for the transport of cosmic-ray particles in both the interstellar and interplanetary media is controlled to a large extent by the interaction of these charged particles with the turbulent electric and magnetic fields of space. Some aspects of this problem have been recently reviewed by JoKIPII (1971), who gives an extensive list of earlier contributors.

There are very few problems of this nature to which one can effect a statistically exact solution and recourse is usually made to some approximate method. One such approximation, the quasilinear, Fokker-Planck approximation, has been used extensively and is discussed in detail in JoKIPII (1971). It would be nice to compare an exact solution with this approximation to provide insight into the realm of validity of the approximation. It is the purpose of this paper to present a statistically exact solution for one special case and to show that the approximation, in this case at least, agrees very well with the exact solution.

\section{A STATISTICALLY EXACT SOLUTION}

Consider the evolution of a distribution of charged particles of charge $e$, momentum $\mathbf{p}$, and rest mass $m$ per particle, under a given, but random, magnetic field $B(t)$ which points in the $z$-direction and is a random function of time alone.

Then if $f(\mathbf{x}, \mathbf{p}, t)$ is the number density of particles in the band of values $\mathbf{x}$ to $\mathbf{x}+$ $\mathrm{d} \mathbf{x}, \mathbf{p}$ to $\mathbf{p}+\mathrm{d} \mathbf{p}$ at time $t$, we have from Liouville's equation that

$$
\frac{\partial f}{\partial t}+v_{i} \frac{\partial f}{\partial x_{i}}+e c^{-1} \varepsilon_{i j k} \delta_{k z} v_{j} B(t) \frac{\partial f}{\partial p_{i}}=0,
$$

where the electric fields (associated with the time variation of $B(t)$ ) are ignored. One can, of course, object that neglect of the electric fields is not permitted in a physically

* Alfred P. Sloan Foundation Fellow. 
realizable problem and we agree with such objections. However, that in no way detracts from the fundamental mathematical problem which is succinctly portrayed by equation (1). Given equation (1) what is its statistically exact solution, and does the quasilinear Fokker-Planck approximation give agreement with the exact solution? We shall answer this question.

We first give the statistically exact solution. To proceed write

in equation (1) to obtain

$$
f=\int f(\mathbf{k}, \mathbf{p}, t) \exp (i \mathbf{k} \cdot \mathbf{x}) \mathrm{d}^{3} \mathbf{k}
$$

$$
\frac{\partial f}{\partial t}+i\left[k_{\|} v_{\|}+k_{\perp} v_{\perp} \cos (\psi-\theta)\right] f=-\mathrm{e} B(t)\left(m c^{\xi}\right)^{-1} \frac{\partial f}{\partial \theta}
$$

where $\xi=\left(1+p^{2} / m^{2} c^{2}\right)^{1 / 2}, \quad v_{\perp}=p_{\perp} /(m c \xi), p_{x}=p_{\perp} \cos \theta, p_{y}=p_{\perp} \sin \theta, k_{x}=$ $k_{\perp} \cos \psi, k_{y}=k_{\perp} \sin \psi, k_{\|}=\bar{k}_{z}, v_{\|}=v_{z}$.

Next cast (3) into dimensionless form. Let $\left\langle B^{2}\right\rangle^{1 / 2}=\varepsilon$, and let the correlation time of $B$ be $T$. Let $B=\varepsilon b, t=T \tau$, so that both the r.m.s. value and correlation time of $b$ are unity. Let $K_{\|}=k_{\|} T, K_{\perp}=k_{\perp} T, \Omega=T e \varepsilon / m c\left(1+p^{2} / m c^{2}\right)^{1 / 2}$. Then equation (3) becomes

$$
\frac{\partial f}{\partial \tau}+i\left[K_{\|} V_{\|}+K_{\perp} V_{\perp} \cos (\psi-\theta)\right] f=-\Omega b \frac{\partial f}{\partial \theta} .
$$

For simplicity we consider the case $K_{\perp} V_{\perp} \sim 0$ (long wavelength normal to $B$ ). Define the new function

Then $g$ satisfies

$$
g(\mathbf{K}, \mathbf{p}, \tau)=\exp \left(i K_{\|} V_{\|} \boldsymbol{\tau}\right) f(\mathbf{K}, \mathbf{p}, \tau)
$$

$$
\frac{\partial g}{\partial \tau}=-\Omega b(\tau) \frac{\partial g}{\partial \theta}
$$

The general solution to equation (6) is

$$
g(\tau, \theta)=H\left(\theta-\Omega \int_{0}^{\tau} b\left(t^{\prime}\right) \mathrm{d} t^{\prime}\right)
$$

where $H(\theta)=g(\tau=0, \theta)$ is the initial value, which we will take to be the same for all members of the ensemble.

We wish to find an equation for $\langle g\rangle$. Suppose we write

$$
H_{0}(\theta)=\sum_{n=-\infty}^{\infty} \mathrm{e}^{i n \theta} h_{n},
$$

where the $h_{n}$ are given constants. Then

$$
\langle g\rangle=\sum_{n=-\infty}^{\infty} h_{n} e^{i n \theta}\left\langle\exp \left[-i n \Omega \int_{0}^{\tau} b\left(t^{\prime}\right) \mathrm{d} t^{\prime}\right]\right\rangle
$$

Now, if $B$ has Gaussian statistics, ${ }^{*}$ we have the identity (see e.g. KuBo (1963); ROSENBLOOM (1954); BOURRET (1965)).

$$
\left\langle\exp \left[-i n \Omega \int_{0}^{\tau} b\left(t^{\prime}\right) \mathrm{d} t^{\prime}\right]\right\rangle=\exp \left\langle-n^{2} \Omega^{2} \int_{0}^{\tau}\left(\tau-t^{\prime}\right) R\left(t^{\prime}\right) \mathrm{d} t^{\prime}\right\rangle
$$

* The reader may wish to consider a situation where Gaussian statistics is not assumed. We show how to handle such a case in Appendix A. 
where $R\left(t^{\prime}\right)=\left\langle b(t) b\left(t+t^{\prime}\right)\right\rangle$. Hence we have the statistically exact solution (for $\left.K_{\perp} V_{\perp} \ll 1\right)$ valid for all times $t>0$,

$$
\langle g\rangle=\sum_{n=-\infty}^{\infty} h_{n} \exp \left[i n \theta-n^{2} \Omega^{2} \int_{0}^{\pi}\left(\tau-t^{\prime}\right) R\left(t^{\prime}\right) \mathrm{d} t^{\prime}\right] .
$$

By inspection, $\langle g\rangle$ satisfies the differential equation

$$
\begin{aligned}
\frac{\partial\langle g\rangle}{\partial \tau} & =\Omega^{2}\left[\int_{0}^{\tau} R\left(t^{\prime}\right) d t^{\prime}\right] \frac{\partial^{2}\langle g\rangle}{\partial \theta^{2}} \\
& =\Omega^{2} D \partial^{2}\langle g\rangle / \partial \theta^{2}
\end{aligned}
$$

exactly, where $D(\tau)=\int_{0}^{\tau} R\left(t^{\prime}\right) \mathrm{d} t^{\prime}$.

Clearly, for $\tau \gg 1$ (the correlation time), this is of the Fokker-Planck form,

$$
\frac{\partial\langle g\rangle}{\partial \tau}=\Omega^{2} D_{\infty} \frac{\partial^{2}\langle g\rangle}{\partial \theta^{2}},
$$

where $D_{\infty}=\int_{0}^{\infty} R\left(t^{\prime}\right) \mathrm{d} t^{\prime}$. Obviously $\langle f\rangle$ satisfies

$$
\frac{\partial\langle f\rangle}{\partial \tau}+i K_{\|} V_{\|}\langle f\rangle=\Omega^{2} D \frac{\partial^{2}\langle f\rangle}{\partial \theta^{2}},
$$

which is the exact evolutionary equation for $\langle f\rangle$. For the special form of the two-point correlation function

$$
R\left(t^{\prime}\right)=\exp \left[-\left|t^{\prime}\right|\right]
$$

equation (13) takes on the explicit form

$$
\frac{\partial\langle f\rangle}{\partial \tau}+i K_{\|} V_{\mathbb{\|}}\langle f\rangle=\Omega^{2}\left(1-\mathrm{e}^{-\tau}\right) \frac{\partial^{2}\langle f\rangle}{\partial \theta^{2}} .
$$

\section{QUASI-LINEAR, FOKKER-PLANCK APPROXIMATION}

Having obtained the exact evolutionary equation (13) for $\langle f\rangle$ in the limit that $K_{\perp} V_{\perp} \ll i$ we now compare this with the usual Quasi-linear, Fokker-Planck (QFP) Approximation. Begin again with equation (1) with $k_{\perp}=0$, and in dimensionless form,

$$
\frac{\partial f}{\partial \tau}+i K_{\|} V_{\|} f=-\Omega b \frac{\partial f}{\partial \theta},
$$

where the dimensionless variables are as used above in Section 2. Let $f=\langle f\rangle+\delta f$, with $\langle\delta f\rangle=0$. The ensemble average of equation (15) is then

$$
\frac{\partial\langle f\rangle}{\partial \tau}+i K_{\|} V_{\|}\langle f\rangle=-\Omega \frac{\partial}{\partial \theta}\langle b(\tau) \delta f\rangle
$$

and subtraction of equation (16) from equation (15) yields

$$
\frac{\partial \delta f}{\partial t}+i K_{\|} V_{\|} \delta f=-\Omega \frac{\partial}{\partial \theta}[b(\tau)\langle f\rangle+b \delta f-\langle b \delta f\rangle] .
$$


Note that equations (16) and (17) are exact. The next step in QFP is to neglect the second order terms $b \delta f-\langle b \delta f\rangle$, and to write the solution to equation (17) in the form

$$
\begin{aligned}
\delta f=-\Omega \int_{\tau_{0}}^{\tau} \mathrm{d} \tau^{\prime} b\left(\tau^{\prime}\right) \frac{\partial}{\partial \theta}\left\langle f\left(\tau^{\prime}, \theta\right)\right\rangle & \exp \left\{i\left(\tau^{\prime}-\tau\right) K_{\|} V_{\|}\right\} \\
& +\delta f\left(\tau_{0}, \theta\right)+[\text { terms of higher order in } \Omega] .
\end{aligned}
$$

Equation (18) is only a good approximation for $\tau-\tau_{0}$ small enough that the zeroorder trajectory (a straight line) is unperturbed in the time $\tau-\tau_{0}$. Alternatively one may show by iteration that the terms of next higher order in $b$ are of order $\Omega\left(\tau-\tau_{0}\right)$ times those retained. Hence, we require

$$
\Omega\left(\tau-\tau_{0}\right) \ll 1 .
$$

The next step in QFP is to substitute the approximate expression (18) into equation (16). One obtains

$$
\begin{aligned}
& \frac{\partial\langle f\rangle}{\partial \tau}+i K_{\|} V_{\|}\langle f\rangle \\
& =\Omega^{2} \frac{\partial}{\partial \theta}\left\{\int_{\tau_{0}}^{\tau}\left\langle b(\tau) b\left(\tau^{\prime}\right)\right\rangle \frac{\partial}{\partial \theta}\left\langle f\left(\tau^{\prime}, \theta\right)\right\rangle \exp \left[i K_{\|} V_{\|}\left(\tau-\tau^{\prime}\right)\right] \mathrm{d} \tau^{\prime}\right\}-\Omega \frac{\partial}{\partial \theta}\left\langle b(\tau) \delta f\left(\tau_{0}, \theta\right)\right\rangle
\end{aligned}
$$

One next assumes that $\Omega$ is small enough that even though (19) is satisfied one can still choose $\tau-\tau_{0}$ much greater than the coherence time of $b(\tau)$, which is unity. Hence, we suppose

$$
\left|\tau-\tau_{0}\right| \gg 1 .
$$

Clearly, for (19) and (21) to be consistent, we require $\Omega \ll 1$. In this limit $\left\langle b(\tau) \delta f\left(\tau_{0}, \theta\right)\right\rangle=0$ and the limit $\tau_{0}$ on the integral in equation (20) may be taken to $-\infty$ since the integral is independent of $\tau_{0}$. Finally, we note that if $\langle f\rangle$ is slowly varying with both $t$ and $\theta$ over the correlation time of $b(t)$ then we can approximate $\left\langle f\left(t^{\prime}, \theta\right)\right\rangle \exp \left[i K_{\|} V_{\|}\left(t-t^{\prime}\right)\right]$ by $\langle f(\tau, \theta)\rangle$ and equation (20) then takes on the standard form

$$
\begin{aligned}
\frac{\partial\langle f\rangle}{\partial \tau} & +i K_{\|} V_{\|}\langle f\rangle=\Omega^{2}\left[\int_{-\infty}^{\tau}\left\langle b(\tau) b\left(\tau^{\prime}\right)\right\rangle \mathrm{d} \tau^{\prime}\right] \frac{\partial^{2}\langle f\rangle}{\partial \theta^{2}} \\
& =\Omega^{2} D_{\infty} \frac{\partial^{2}\langle f\rangle}{\partial \theta^{2}}
\end{aligned}
$$

where $D_{\infty}$ is often termed a "Fokker-Planck" coefficient.

Note that equation (22) is immediately seen to be equal to the exact equation (12) in the limit $\tau \gg 1$. The standard QFP analysis, which has been applied successfully in other situations passes this test as well. This special case, permitting comparison with an exact solution, provides support for the use of the QFP approach in situations where exact solutions are not available.

We note one further, surprising fact. The approximate equation (22) was derived assuming $\Omega \ll 1$, but it is manifestly correct to all orders in $\Omega$ since equation (12) is exact. The neglected terms $b \delta f-\langle b \delta f\rangle$ must cancel in this case for large times. It is 
clearly of great importance to determine whether or not this cancellation occurs in other cases.

Acknowledgements-This work was supported, in part (J. R. J.) by NASA under Grant NGR 05 . 002-160, and in part (I, L.) by the United States Air Force, Air Force Systems Command under contract F-19628-72-C-0056.

\section{REFERENCES}

Bourret R. C. (1965) Car. J. Phys, 43, 619.

ChandrasekHaR S. (1943) Rev. mod. Phys. 15, 1.

JokIPII J. R. (1971) Rev. geophys. Space Phys. 9, 27.

Kubo R. (1963) J. math. Phys, 4, 174.

Rosenbloom A. (1954) in Proc. PIB Symp. Information Networks, p. 145, Polytechnic Institute of Brooklyn, New York.

UhleNBeCK G. E. and ORnstein L. S. (1930) Phys. Rev. 36, 823.

\section{APPENDIX A}

Here we are interested in the evolution of $f$ knowing that at $t=0$ the magnetic field takes on the value $b_{0}$, and at any later time the probability of finding a given strength magnetic field evolves according to a prescribed rule.

To proceed write

in equation (3) to obtain

$$
f=\sum_{n=-\infty}^{\infty} A_{n}(t) \exp (i n \theta)
$$

$$
\frac{\mathrm{d} A_{n}}{\mathrm{~d} t}=-i\left[k_{k} v_{\|} A_{n}+\frac{1}{2} v_{\perp} k_{\perp}\left(A_{n+1}+A_{n-1}\right)\right]-i e n B(t) A_{n} /(m c \xi) .
$$

Now $B(t)$ is random with zero mean. Again let the r.m.s. value of $B$ be $\varepsilon$ and let the correlation time of $B$ be $T$. Then write $T_{\tau}=t, B(t)=\varepsilon b(\tau)$ so that the correlation time of $b(\tau)$ is initity, and its r.⿲丿丨. value is also unity. Then equation (A.2) becomes

$$
\frac{\mathrm{d} A_{n}}{\mathrm{~d} \tau}=-i n \Omega b A_{\mathrm{s}}-i\left[K_{! !} V_{\|} A_{n}+\frac{1}{2} V_{\perp} K_{\perp}\left(A_{n+1}+A_{n-1}\right)\right],
$$

where $K_{\|}=k_{\|} T, K_{\perp}=k_{\perp} T, \Omega=T e \varepsilon /(m c \xi)$.

Let $P$ be the probability of finding the set of values $\left\{A_{n}\right\}$ and the value $b$ at time $\tau$. Further let $\mathscr{L}(b)$ denote the probability operator field of finding the value $b$ at time $\tau$ on its own. Then from the general theory of stationary random processes (see e.g. UHLENBECK and ORNSTEIN (1930); CHANDRASEKHAR (1943)) we have

$$
\frac{\partial P}{\partial \tau}=\mathscr{L}(b) P+\sum_{n=-\infty}^{\infty} \frac{\partial}{\partial A_{n}}\left\{\left\{P\left[\left[i n \Omega b A_{n}+i\left[K_{\|} V_{\mathrm{I}} A_{n}+\frac{1}{2} V_{\perp} K_{\perp}\left(A_{n+1}+A_{n-1}\right)\right]\right]\right\},\right.\right.
$$

subject to the initial conditions

$$
P(\tau=0)=\delta\left(b-b_{0}\right) \prod_{n=-\infty}^{\infty} \delta\left[A_{n}-y_{n}\right],
$$

where $b_{0}$ and the set $\left\{y_{n}\right\}$ are arbitrary.

The immediate task before us is, evidently, to solve equation (A.4) for $P$ and then to compute the ensemble average value of $f$, say $\langle f\rangle$ where

and

$$
\langle f\rangle=\sum_{n=-\infty}^{\infty}\left\langle A_{n}\right\rangle \exp (i n \theta)
$$

$$
\left\langle A_{n}\right\rangle=\int A_{n} P \mathrm{~d} b \prod_{m=-\infty}^{\infty} \mathrm{d} A_{m} .
$$

We have so far been unable to solve equation (A.4) subject to the initial value conditions (A.5). However, in order to obtain an equation from which we can determine $\langle f\rangle$ a complete solution to equation (A.4) is not necessary as we now demonstrate directly. 
Multiply equation (A.4) by $A_{r}$ and then integrate $\prod_{m=-\infty}^{\infty} \mathrm{d} A_{m}$ to obtain

$$
\frac{\partial q_{r}}{\partial \tau}=\mathscr{L}(b) q_{r}-i\left[r \Omega b q_{r}+K_{\|} V_{\|} q_{r}+\frac{1}{2} V_{\perp} K_{\perp}\left(q_{r+1}+q_{r-1}\right)\right],
$$

where

$$
q_{r}=\int A_{r} P \prod_{m=-\infty}^{\infty} \mathrm{d} A_{m}
$$

By inspection we see that in order to obtain $\left\langle A_{r}\right\rangle$ all we need to find is $q_{r}$ for then

$$
\left\langle A_{\tau}\right\rangle=\int q_{r} \mathrm{~d} b
$$

When the probability of finding $b$ on its own at time $\tau$ is described by the ORNSTEIN-UHLENBECK (1930) process (and we shall assume that it is for the remainder of this appendix) then

$$
\mathscr{L}(b) P=\frac{\partial}{\partial b}(b P)+\frac{\partial^{2} P}{\partial b^{2}} .
$$

Equation (A.8) can then be solved exactly subject to the initial conditions

$$
q_{r}(\tau=c)=y_{r} \delta\left(b-b_{0}\right)
$$

as we shall now demonstrate.

To proceed with the solution use equation (A.11) in equation (A.8) and then write

$$
b=\xi \exp (-\tau), \quad g_{r}=g_{r} \exp \left[\tau\left(1-i K_{\|} V_{i \mid}\right)\right],
$$

when equation (A.8) becomes

$$
\frac{\partial g_{r}}{\partial \tau}=\exp (2 \tau) \frac{\partial^{2} g_{r}}{\partial \xi^{2}}-i r \Omega \xi_{r} \exp (-\tau)-\frac{1}{2} i V_{\perp} K_{\perp}\left(g_{\tau+i}+g_{\tau-1}\right),
$$

subject to the initial condition

$$
g_{r}(\tau=0)=y_{r} \delta\left(\xi-b_{0}\right) .
$$

To effect a solution to equation (A.14) it is opportune to Fourier transform in $\xi$-space with

$$
g_{r}(\tau, \xi)=\int_{-\infty}^{\infty} G_{r}(\tau, \mu) \exp (i \mu \xi) \mathrm{d} \mu,
$$

where $G_{r}$ satisfies the equation

$$
\frac{\partial G_{r}}{\partial \tau}=-\mu^{2} e^{2 \tau} G_{r}-\frac{1}{2} i V_{\perp} K_{\perp}\left(G_{r+1}+G_{r-1}\right)-r \Omega e^{-\tau} \partial G_{r} / \partial \mu
$$

subject to the initial condition

$$
G_{\tau}(\tau=0)=y_{r} \exp \left(-\mu b_{0}\right)
$$

Now multiply equation (A.17) by exp $(i r \beta)$ and sum over $r$ to obtain

$$
\frac{\partial S}{\partial \tau}=-\mu^{2} e^{2 \tau} S-i V_{\perp} K_{\perp} S \cos \beta+i \Omega e^{-\tau} \frac{\partial^{2} S}{\partial \mu \partial \beta},
$$

where

and

$$
S=\sum_{r=-\infty}^{\infty} G_{r} \exp (i r \beta), \quad S(\tau=0)=\sum_{r=-\infty}^{\infty} y_{r} \exp \left(-i \mu b_{0}+i r \beta\right)
$$

Now write

$$
G_{r}(\tau, \mu)=\left(2 \pi^{-1}\right) \int_{0}^{2 \pi} S(\beta, \tau, \mu) \exp (-i r \beta) d \beta .
$$

$$
S=U \exp \left(-\frac{\pi}{2} \mu^{2} \exp (2 \tau)-i \tau V_{\perp} K_{\perp} \cos \beta\right),
$$


when equation (A.19) gives

where

$$
\frac{\partial U}{\partial \tau}=i \Omega \exp (-\tau)\left[\frac{\partial Q}{\partial \mu}-\mu \exp (2 \tau) Q\right],
$$

and

$$
Q=\frac{\partial U}{\partial \beta}+i V_{\perp} K_{\perp} \tau \cos \beta U
$$

$$
U(\tau=0)=\exp \left(\mu^{2} / 2\right) \sum_{r=-\infty}^{\infty} y_{r} \exp \left(i r \beta-i \mu b_{0}\right),
$$

When $K_{\perp}=0$ equation (A.23) is readily solved subject to the initial value (A.25) and then

$$
\begin{aligned}
G_{r}\left(K_{\perp}=0\right)=y_{r} \exp \left[-\frac{1}{2} \mu^{2} \exp (2 \tau)\right. & +\frac{1}{2}(\mu+r \Omega \exp (-\tau))^{2} \\
& \left.-\left(r \Omega+i b_{0}\right)(\mu+r \Omega \exp (-\tau))+r \Omega\left(i b_{0}+\frac{1}{2} r \Omega\right)\right] .
\end{aligned}
$$

Use of equation (A.26) in equations (A.13), (A.16) and (A.10) then gives

$$
\left\langle A_{r}\left(K_{\perp}=0\right)\right\rangle=y_{r} \exp \left\{-i \tau K_{\|} V_{\|}+i b_{0} r \Omega[1-\exp (-\tau)]\right.
$$

And then we have

$$
\left.-r^{2} \Omega^{2}\left[\left[\tau+\exp (-\tau)-\frac{1}{2}[1+\exp (-2 \tau)]\right]\right]\right\}
$$

$$
\left\langle f\left(K_{\perp}=0\right)\right\rangle=\sum_{n=-\infty}^{\infty}\left\langle A_{n}\right\rangle \exp [i n(\psi-\varphi)] .
$$

At $\tau=0$ let the value of $\langle f\rangle$ be $f_{0}\left(\varphi, p_{\perp}, p_{\|}, K_{\|}\right)$.

Then

$$
y_{\tau}=(2 \pi)^{-1} \int_{0}^{2 \pi} f_{0}\left(\varphi^{\prime}, p_{\perp}, p_{\|}, K_{\|}\right) \exp \left[i r\left(\varphi^{\prime}-\psi\right)\right] \mathrm{d} \varphi^{\prime} .
$$

Use of equations (A.27) and (A.29) in equation (A.28) then gives the statistically exact solution (for $K_{\perp}=0$ ) as

$$
\begin{aligned}
\langle f\rangle=(2 \pi)^{-1} \exp (- & \left.\tau K_{\|} V_{\|}\right) \sum_{r=-\infty}^{\infty} \int_{0}^{2 \pi} \mathrm{d} \varphi^{\prime} f_{0}\left(\varphi^{\prime}, p_{\perp}, p_{\|}, K_{\|}\right) \\
& \times \exp \left\{i r\left[\varphi^{\prime}-\varphi\right]+i b_{0} \Omega\left(1-e^{-\tau}\right)-r^{2} \Omega^{2}\left[\tau+e^{-\tau}-\frac{1}{2}\left(1+e^{-2 \tau}\right)\right]\right\} .
\end{aligned}
$$

Equation (A.30) is valid for all values of $\tau \geq 0$.

It is well known that

$$
\sum_{r=-\infty}^{\infty} \exp \left(i r \alpha-r^{2} \gamma^{2}\right)=\pi^{1 / 2} \gamma^{-1} \sum_{n=-\infty}^{\infty} \exp \left[-\frac{1}{4} \gamma^{-2}(2 \pi n-\alpha)^{2}\right]
$$

so that we can write equation $(\hat{A} .30)$ in the form

$$
\begin{aligned}
\left\langle f\left(K_{\perp}=0\right)\right\rangle=\exp & \left(-i \tau K_{\|} V_{\|}\right)(2 \Omega)^{-1}\left\{\pi\left[\tau+e^{-\tau}-\frac{1}{v}\left(1+e^{-2 \tau}\right)\right]\right\}^{1 / 2} \\
& \times \sum_{n=-\infty}^{\infty} \int_{0}^{2 \pi} \mathrm{d} \varphi^{\prime} f_{0}\left(\varphi^{\prime}, p_{\perp}, p_{\|}, K_{\|}\right) \exp \left\{-\frac{1}{4} \Omega^{-2}\right. \\
& \left.\times\left[2 \pi n+\varphi-\varphi^{\prime}-b_{0} \Omega(1-\exp (-\tau))\right]^{2}\left[\tau+e^{-\tau}-\frac{1}{2}\left(1+e^{-2 \tau}\right)\right]^{-1}\right\} .
\end{aligned}
$$

For $\tau \gg 1$ equation (A.32) has the form of a diffusive behavior for $\langle f\rangle$ in the phase angle $\varphi$.

We again point out that equation (A.32) is statistically exact (for $K_{\perp}=0$ ) and describes the evolution in phase angle $\varphi$ of the average $\langle f\rangle$ for all time starting from a given, but arbitrary, initial average state $f_{0}$.

It is also clear by inspection of equation (A.32) that $\langle f\rangle$ satisfies the statistically exact differential equation

$$
\frac{\partial\langle f\rangle}{\partial \tau}=-i K_{\|} V_{1}\langle f\rangle-i b_{0} \Omega e^{-\tau} \frac{\partial\langle f\rangle}{\partial \varphi}+\Omega^{2}\left[1-e^{-\tau}+e^{-2 \tau}\right] \frac{\partial^{2}\langle f\rangle}{\partial \varphi^{2}} .
$$

For $\tau \gg 1$ this reduces to equation (14) indicating that even for evolving statistical distributions of fields the quasilinear Fokker-Planck approach gives the correct average equation in the long time limit. 